\title{
LE CHOIX DES ANNALES
}

BACHTIN (Michail M.), Formen der Zeit im Roman Untersuchungen zur historischen Poetik, Francfort-sur-le-Main, Fischer Wissenschaft, 1989, 251 p.

Édition en livre de poche de la traduction allemande (1986) du livre où Bachtin a résumé sa dernière idée du concept de chronologos, outil de recherche sur les rapports temps-espace dans l'histoire, le folklore et la littérature. A quand une traduction française?

BERDING (Helmut), FRANCOIS (Étienne), ULLMANN (Hans-Peter), La Révolution, la France et l'Allemagne. Deux modèles opposés de changement social, Paris, Éditions de la Maison des Sciences de l'Homme, 1989, 424 p.

Une tentative résolue comparée qui mérite d'être saluée. La Révolution est, à vrai dire, secondaire dans cette bonne série d'études qui proposent surtout de suivre les évolutions économiques, sociales et culturelles des deux mondes dans le cadre d'une chronologie élargie (1770-1820) et en prenant en compte la diversité des expériences allemandes.

DUHOT (Jean-Joël), La conception stolcienne de la causalité, Paris, Librairie Vrin, 1989, 302 p.

Aucune des écoles philosophiques antiques n'a connu le couple causeeffet. Reprenant le système ternaire de Platon : élément actif, élément passif, produit, et écartant les quatre causes d'Aristote (matérielle, motrice, formelle, finale) les stoiciens reconnaissent trois types de causes : externe (sans importance fondamentale), interne (les dispositions de l'élément récepteur), et enfín une cause dite liée, c'est-à-dire l'altération du souffle qui maintient la cohésion de toutes choses. Des questions sur les définitions historiques de la causalité, et des éléments pour une réflexion sur nos propres pratiques.

LAURENT (Donatien), Aux sources du Barzaz-Breiz. La mémoire d'un peuple, Douarnenez, Éditions Ar Men (Le Chasse-Marée), 1989, 335 p.

Superbe édition bilingue du célèbre recueil de chants populaires bretons publiés en 1839 par le jeune chartiste Théodore Hersart de La Villemarqué qui s'enferma toute sa vie dans un silence énigmatique face à ses détracteurs qui l'accusèrent, Macpherson breton, d'avoir forgé ces poèmes. Donatien Laurent a découvert en 1964 les carnets prouvant l'authenticité du fonds de ces textes notés dans des veillées par La Villemarqué et les 
publie avec un commentaire d'un intérêt exceptionnel pour la compréhension des rapports entre culture savante et folklore et le fonctionnement de la mémoire populaire.

"Le christianisme russe entre millénarisme d'hier et soif spirituelle d'aujourd'hui ", Cahiers du Monde russe et soviétique, décembre 1988, numéro double XXIX, 3-4, avec un avant-propos de Georges Nivat.

Les actes du colloque de juin 1988 à Genève évoquent des aspects hétérodoxes ou peu connus du christianisme en Russie depuis le mythe du pape antéchrist jusqu'à la figure religieuse de l'athée russe, adonné à son apostolat.

LOMBARD-JOURDAN (Anne), "Montjoie et saint Denis / "Le centre de la Gaule aux origines de Paris et de Saint-Denis, Paris, Presses du Centre National de la Recherche Scientifique, 1989, 392 p.

Une érudition extraordinaire, une capacité remarquable à faire converger toutes les sciences auxiliaires de l'histoire, une étonnante originalité d'esprit, permettent à Anne Lombard-Jourdan d'aller encore plus loin dans le renouvellement de l'histoire du destin antique et médiéval de Paris.

MACHEREL (Claude) et STEINAUER (Jean), L'État de ciel, Portrait de ville avec rite. La féte-Dieu de Fribourg, Fribourg (Suisse), Méandre Éditions, diffusion Paris, Desclée de Brouwer, 1989, 328 p. et 32 p. d'héliogravures hors texte.

Ethnologie, histoire, écriture, iconographie ont rivalisé de savoir, d'intelligence et de bonheur pour éclairer une fête exemplaire depuis le Moyen Age. Un livre en état de grâce sur les rapports entre une ville et le ciel.

Revue économique, volume 4, numéro 2, mars 1989, "L'économie des conventions $)$.

Où il est démontré que des emprunts aux sciences sociales voisines permettent à l'économie de remettre en cause l'un de ses paradigmes dominants : l'existence d'un marché concurrentiel et universel parfait. La notion de convention définie à l'intersection d'un cadre contraignant et des pratiques individuelles permet de voir dans l'échange marchand une forme parmi d'autres de la coordination sociale et ouvre les possibilités d'une histoire. Ce dernier axe peu exploré ici. Mais un bon exemple des révisions aujourd'hui en cours dans les sciences sociales.

VERNANT (Jean-Pierre), L'individu, la mort, l'amour. Soi-même et l'autre en Grèce ancienne, Paris, Éditions Gallimard, "Bibliothèque des Histoires ", 1989, 232 p.

Dans une société où l'on vit sans cesse sous le regard d'autrui, qui exalte l'individu mais ignore la personne, comment faire de l'autre soi-même? Une triple enquête sur la figure des dieux, le masque de la mort, le visage de l'être aimé. Plus que jamais Jean-Pierre Vernant nous montre que la société et la pensée grecques sont le terrain modèle de l'anthropologie historique.

WHITE (Stephen D.), Custom, Kinship and Gifts to Saints. The Laudatio Parentum in Western France (1050-1150), Chapel Hill-Londres, The University of North Carolina Press, 1988, 313 p.

Comme l'a écrit Caroline Bynum " un livre qui défie les opinions recues sur le droit, le pouvoir et la famille ainsi que la bonne utilisation de l'analyse statistique et des modèles anthropologiques en histoire ". Une nouvelle façon d'étudier et de comprendre la société féodale. 


\section{nouveauté}

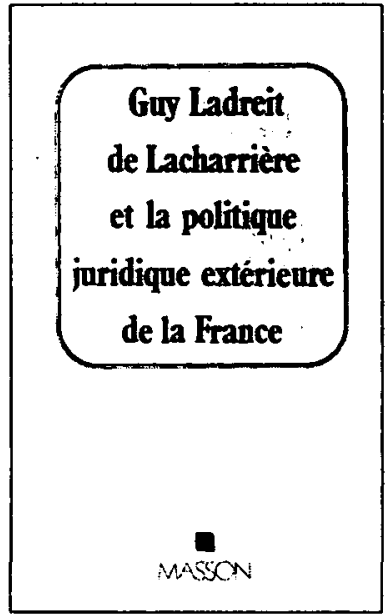

Cet ouvrage, publie en l'honneur de la mémoire de Guy Ladreit de Lacharrière, comporte trois parties.

La première est un hommage rendu à celui-ci, la seconde retrace sa carrière et analyse son œuvre,

la troisieme regroupe des contributions qui se concentrent sur ses grands domaines d'activité :

la théorie générale du droit international, le droit de la mer, le droit du développement, les problèmes de sécurité, les questions juridictionnelles et la diplomatie multilatérale.

1989, 432 pages, $195 \mathrm{~F}$

(Prix public T.T.C. au 01.05.89) 


\title{
PROMETEO
}

\author{
Arnoldo Mondadori Editore \\ Rivista trimestrale di scienze e storia \\ Direttore scientifico: Valerio Castronovo
}

Comitato scientifico: M. Augé, M. Aymard, J. Beck, D. Bovet, P. Burke, A. Carotenuto, V. Castronovo, N. Chomsky, A. Danchin, M. Detienne, U. Eco, I. Eibl-Eibesfeldt, P.K. Feyerabend, L. Gambi, F.M. Gil, G. Giorello, M. Godelier, H.E. Gombrich, J. Goody, F. Héritier, A. Hirschman, G. Holton, A. Jacquard, J. Kocka, J.D. Laioux, V. Lanternari, J. Le Goff, R.C. Lewontin, N. Lubmann, C. Magris, G.L. Mosse, W.H. Newton-Smith, A Oliverio, A Piatigorsky, C. Poni, T. Regge, J. Revel, I. Sachs, G.W. Stocking jr., V. Strada, P. Sylos Labini, K. Thomas, V. Valeri, N. Wachtel.

Anno VII n. 26

Giugno 1989

Tullio Regge: Asteroidi in libertà

Adolf Grünbaum: La cattiva scienza

Vittorio Strada: I costruttori di Dio

Michel Mollat: L'ambiguità dei poveri

Robert S. Root-Bernstein: La scoperta e il caso

Marco Meriggi: Modelli di borgbesia

Marcello Massenzio: L'animale e il rito

Pepa Sparti: La torre del progresso

Giuseppe Berta: Il capitalismo dei gentlemen

Franca Zoccoli: Le geometrie della trapunta

Renate B. Viertler: Cosmologia bororo

Hans Peter Duerr: Nudità e pudore

Note e rassegne:

Utopie: Giorgio Celli - La scimmia, immagine povera dell'uomo

Cosmologie: Angioletta Coradini - L'origine del sistema solare

L'immaginario matematico: Berkelius - Il numero: origini e motivi

Tecnologie: Franco Tinti - Molte leghe più avanti

Variazioni: Viva Tedesco - Effetto a sorpresa per ascoltatori complici

Itinerari: Francesco Iengo - Scrittori tra la folla

Abbonamento annuale per l'italia:

Abbonamento annuale per l'estero:

Live 32.000 anziché Lire 40.000

L'ordine va inviato insieme al pagamento a:

Amoldo Mondadori Editore Servizio Abbonamenti 20090 - Segrate (MI) Italia

Taniffe valide per il 1989 


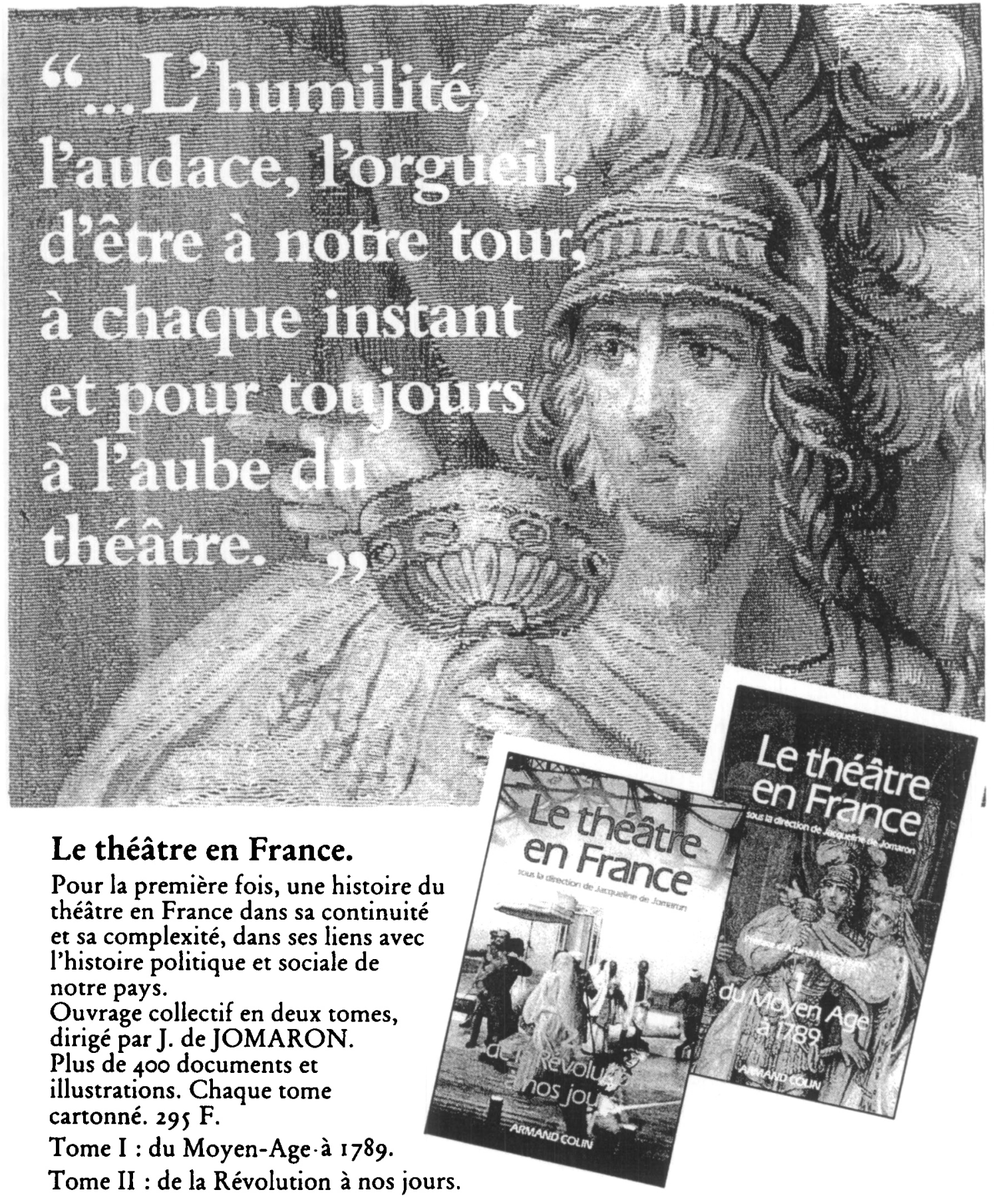




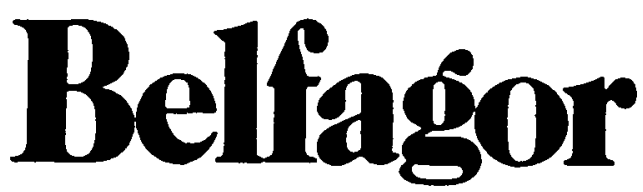

rassegna di varia umanità

diretta da Carlo Ferdinando Russo

Sommario del fascicolo II

ANNO XLIV

31 MARZO 1989

SAGGI E STUDI

Marcello Massenzio: Ernesto De Martino: l'aspetto critico della sessualità con un saggio inedito . . . . . . Pag.

Arnaldo Pizzorusso: Sui "Cabiers» di Valéry: le immagini del soggetto

RITRATTI CRITICI DI CONTEMPORANEI

Sergio Turone: Mario Bonescbi

VARIETA E DOCUMENTI

Marino Raicich: Il caso Manzoni . . . . . . . . . $\quad 167$

BRUNO WANROOIJ: Umberto Notari, o dell'ambigua modernitd . . . 181

BRUNo PIschedDA: Istoriar romanzi . . . . . . . . . . 194

NOTERELLE E SCHERMAGLIE

MAssimo Aloisi: Un fantasmo collocato a riposo . . . . . . 203

Gianmatteo del Brica: Lettera pendolare. . . . . . . 210

Massimo Mugnar: In difesa dell'Accademia delle Scienze e delle Arti di Berlino . . . . . . . . . . . . . .

RECENSIONI

MARY Grbson, Prostitution and the State in Italy, 1860-1915 (Renzo Villa). . . . . . . . . . . . . . .

Wilhelm PötTers, Cbi era Laura? Strutture linguistiche e matematicbe nel "Canzoniere " di Francesco Petrarca (Joseph A. Barber) . . 229

Arthur Schnitzler, Opere, a cura di Giuseppe Farese (Sotera Fornaro) 231

Anco MArzio MutTterle, Il professore ombroso. Quattro studi su Giacomo Zanella (Carlo A. Madrignani) . . . . . . . . . 236

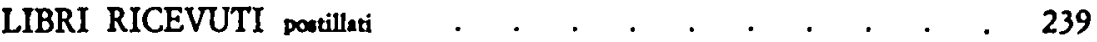

Croce $i$ crociani e gli enticraciani . . . . . . . . . . . 166

Sede: «La Belfagoriana presso Casa editrice Leo S. Olschki 50100 Firenze Indirizzare manoscritti corrispondenza libri alla Direzione in Bari: casella postale 29170100 Bari Tel. (080) 540.065

Direzione e redazione: C.F. Russo Giacomo Annibaldis Francesco De Martino

Sotera Fornaro Marzia Pieri Claudio Pogliano Onofrio Vox

Abbonamento annuo Lire 46.000 (est. Lire 70.000) - Sostenitore Lire 200.000

Un fascicolo Lire 15.000 (est. Lire 19.000) - C.c.p. 21920509 - *Belfagot * Firenze Amministrazione:

Casa editrice Leo S. Olschki - Casella postale 6650100 Firenze Tel. (055) 65.30 .684 


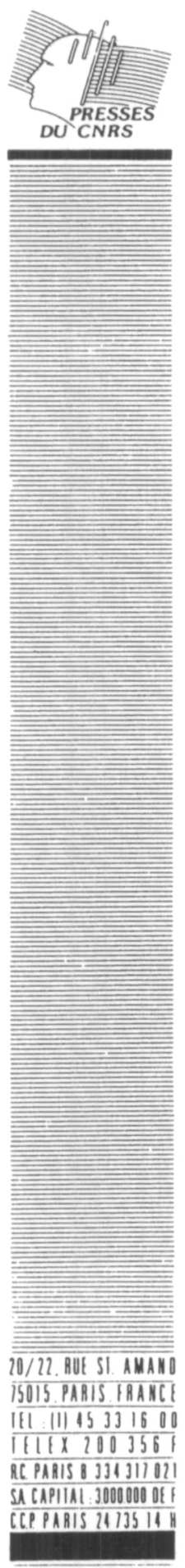

Aux Éditions du CNRS

\section{PALÉORIENT}

Revue pluridisciplinaire de préhistoire et de protohistoire de I'Asie du Sud-Ouest

$14 / 1-1988$

Au sommaire notamment :

- Archéologie du Xinjiang des origines aux Han (1'0 partie) par C. DEBAINE-FRANCFORT;

- Céramiques de Sarazm (Tadjikistan, URSS) : problèmes d'échanges et de peuplement à la fin du chalcolithique et au début de l'Age du bronze par A. ISAKOV et B. LYONNET;

- Études physico-chimiques des matières premières et production métallurgique à l'Age du Bronze au Kazakhstan central par E. F. KOUZNETSOVA, N. F. PCHENITCHNAIA et E. N. SOULEIMENOV.

$21 \times 30-184$ pages

Prix : $200 \mathrm{~F}$

ISBN 2-222-04249-6

\section{PALÉORIENT EST EN COMMANDE PERMANENTE}

\section{BON DE COMMANDE}

à compléter et à retourner aux Presses du CNRS, 20-22 rue Saint-Amano 75015 Paris

Prénom …................................................. Nom

Profession

No

Rue

Commune

Code Postal Bureau distributeur Pays

$\square$ Souhaite recevoir exemplaires de Paléorient 14/1 - 1988 au prix de $200 \mathrm{~F}$. Ci-joint mon règlement de francs (ajouter $15 \mathrm{~F}$ pour frais de port)

- Souhaite s'inscrire pour recevoir Paléorient en commande permanente Date :

Signature :

code : 


\section{Quaderni di storia}

Anno XV, numero 29 / gennaio-giugno 1989

\section{Saggi}

Diego LANza, Una ragazza, offerta al sacrificio... 5

E. Badian, Introduction to Diels and Wilamowitz 23

Hermann Diels, The Evolution of Greek Pbilosopby 33

UlRich voN Wilamowitz MöllendoRfF, The Development of the Hellenic Spirit

Michele Bernardini, L'esposizione di tessuti durante importanti cerimonie nel periodo timuride ( $X V$ sec.)

Carles Mtralles, la tradizione giambica

Miscellanea

WILLIAM M. CALDER III, The Members of -Wilamowitz' Graeca

Francesca Anglo, Etica aristocratica ed azione politica in Crizia

GIUSEPPE VELARDt, «L'azione di attendere»: il partito comunista francese e la questione algerina

Recensioni

A. Momigliano, Pagine ebraiche a cura di S. Berti (Fausto Parente)

J. Andreau, La vie financière dans le monde romain: les métiers de manieurs d'argent (Giorgio Maselli) 179

K. RaAflaub, Die Entdeckung der Freibeit (Vittorio Citti) 189

M.M. SAssi, La scienza dell'uonio nella Grecia antica (Aldo Corcella)

M. BRETONE, Storia del diritto romano (Gianfranco Liberati) 205

Esce 2 gennaio e a luglio. Redazione e Amministrazione: Edizioni Dedalo spa, casella postale 362, 70100 Bari (tel. 080/371555), c/c postale 11639705. Abbonamento 1989 lire 24.000 , estero 30 dollari, un fascicolo lire 15.000 Spedizione in abbonamento postale, gruppo IV, 70\%.

Indirizase corrispondenza, datriloseritti e libri per la Rassegna bibliografica a: Lucia. no Canfora, casella postale 200, 70100 Bari. 


\section{LECHRISIIANISME RUSSE}

\section{entre millénarisme d'hier et soif spirituelle d'aujourd'hui}

Le christianisme russe entre le millénarisme d'hier et la soif spirituelle d' aujourd' hui, recueil des actes d'un colloque organisé par l'Université de Genève en juin 1988, rassemble des textes qui évoquent des aspects peu connus du christianisme en Russie entre le XVII siècle et nos jours. Depuis le mythe du pape-antéchrist, repris par les sectes du XVIII' siècle et présent même dans la propagande bolchevique, jusqu'aux éléments millénaristes dans la révolution de 1917, nous découvrons ici des aspects politiques et culturels d'un christianisme populaire et hétérodoxe qui bouillonna toujours sous l'orthodoxie. Nous retrouvons aussi les tendances pro-catholiques d'une partie de l'aristocratie russe au début du XIX' siècle, et, à l'époque des uradicaux» russes des années 60 et suivantes du siècle demier, l'étonnante figure religieuse de l'athée russe, passionnément adonné à son apostolat.

Sainteté populaire, sens du sacré dans la prose russe du «grand siècle», évolution religieuse de certaines figures importantes de l'émigration d'après 1917, idée d'un socialisme chrétien évoquée dès le début du siècle par un Serge Boulgakov, reprise plus tard par un Georges Fedotov - voici quelques-uns des thèmes étudiés ici également, et qui nous mènent jusqu'à aujourd'hui, où la "soif spirituelle» est si évidente au travers de certains auteurs soviétiques cependant que l'athéisme officiel est à la recherche d'une nouvelle formule. A côté d'universitaires et de chercheurs français, suisses, polonais, israélien, américain et allemand, ont pris part au recueil des personnalités de l'émigration actuelle ainsi que deux grands écrivains contemporains, l'un qui ouvre, l'autre qui conclut le livre: André Siniavski, qui vit à Paris, et Valentin Raspoutine, qui vit à Irkoutsk. Quatre textes russes sont publiés dans l'original, les autres sont soit traduits, soit écrits en français.

Textes et études réunis dans Le christianisme russe entre le millénarisme d' hier et la soif spirituelle d' aujourd' hui font donc le point sur un nombre important de questions concernant l'héritage culturel et social du christianisme en Russie au moment où la Russie vient de fêter le millénaire de son baptême et semble revenir partiellement à cette source première de sa civilisation.

Cahiers du monde russe et soviétique, $\mathrm{XXIX} / 4-190,00 \mathrm{~F}$

Diffusion CID - 131 bd Saint Michel, 75005 Paris 


\title{
REVUE DE L'HISTOIRE DES RELIGIONS
}

Publication trimestrielle fondée en 1880

\author{
COMTTE DE PATRONAGE \\ André BAREAU, André CAQUOT \\ Jean DELUMEAU, Jean-Pierre VERNANT \\ DIRECTION \\ Antoine GUILLAUMONT, Charles AMIEL \\ CONSEIL DE REDACTION \\ Jean BAZIN, Nicole LORAUX \\ Charles MALAMOUD, Maurice OLENDER
}

Au sommaire des fascicules 1 et 2 de 1989

Hérakleiè Nosos. L'épilepsie d'Héraklès (E. Filhol). Le mythe de Néron. De l'Apocalypse de saint Jean au Talmud de Babylone (M. Bodinger). Prières privées en islam traditionnel. Autour d'un texte de Razi (G. Monnot). La conquête de l'Irlande. Religion et migration au XVII ${ }^{\mathrm{e}}$ siècle (B. Cottret).

Le masque du "Phallen". Sur une épiclèse de Dionysos à Méthymna (M. Casevitz, F. Frontisi-Ducroux). L'instauration de la Puissance divine dans l'image du temple en Inde du Sud (G. Colas). Le panthéon de l'Arabie du Sud préislamique : état des problèmes et brève synthèse (J. Ryckmans). Midrach et Byzance. Sur une traduction française du "midrach rabba" (N. de Lange).

Comptes rendus, Notes bibliographiques

Chroniques, Livres reçus.

CORRESPONDANCE

Revue de l'Histoire des Religions

PUF, 12 rue Jean-de -Beauvais, 75005 PARIS

VENTE ET ABONNEMENTS

Presses Universitaires de France

14, avenue du Bois- de-l'Epine

B. P. 90,91003 Evry Cedex

(C.C.P. : PARIS 1302-69 C)

Abonnement 1989 (4 fascicules)

France : $290 \mathrm{~F}$ Etranger : $365 \mathrm{~F}$

Prix au numéro: $90 \mathrm{~F}$ 


\title{
Lumières, utopies, révolutions : espérances de la démocratie
}

\author{
Hommage à Bronislaw Baczko
}

De Rousseau à Solidarnośc, ce volume nous dit avec quelle intensité les hommes travaillent pour la justice, pour la fraternité, pour les libertés. Ce volume est un hommage à un de ces hommes, mais aussi à l'historien sensible aux utopies, aux imaginaires sociaux, à la révolution; à l'historien chez lequel l'histoire sociale des idées s'élargit aux dimensions d'une anthropologie politique qui reprend en termes nouveaux des questions fondamentales, en prise sur les problèmes de notre société.

\section{OFFERT EN SOUSCRIPTION jusqu'au 30 septembre 1989}

au prix de 40 Fr. s.

LIBRAIRIE DROZ S.A. - 11, rue Massot - GENÈVE 


\section{Le genre humain}

revue publiée par les éditions du Seuil

\section{COMITÉ}

Marc Augé

Jean Bernard

François Jacob

Jacques Le Goff

Léon Poliakov

Jean-Pierre Vernant
DIRECTION

Maurice Olender

Ecole des Hautes Etudes

en Sciences Sociales

54 , boulevard Raspail

bureau 928

75270 Paris Cedex 06

Tél. 45.44.39.79 (p. 421)
RÉDACTION

Lydia Flem

Nadine Fresco

Yves Hersant

Albert Jacquard Jean-Marc Lévy-Leblond

Maurice Olender

Léon Poliakov

Alain Schnapp

Emmanuel Terray

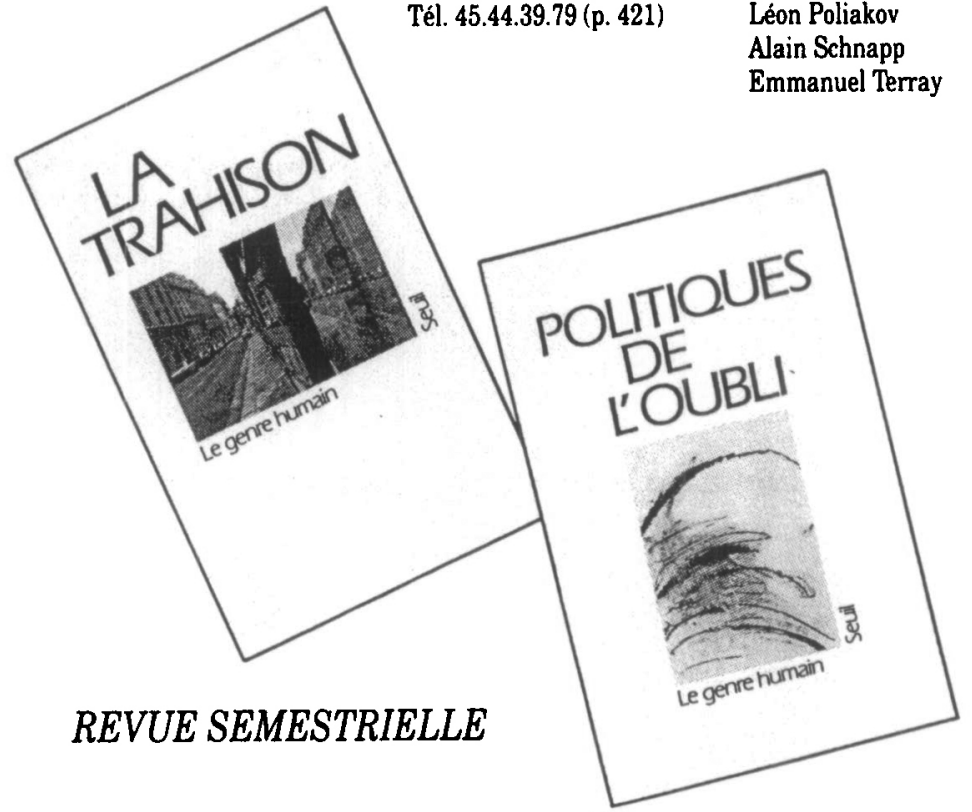

No 18 - Politiques de l'oubli

No 16-17 - La trahison

$85 \mathrm{~F} \quad \mathrm{~N}^{\circ} \mathbf{9} \cdot 1984$ ?

$79 \mathrm{~F}$

$\mathrm{N}^{\circ} 15$ - La fièvre

$99 \mathrm{~F}$

$79 \mathrm{~F}$

$N^{\circ} 14$ - La valeur

$79 \mathrm{~F}$

№ 13 - Lamour

$79 \mathrm{~F}$

$\mathrm{N}^{\circ} 12$ - Les usages de la nature $79 \mathrm{~F}$

$^{\circ} 11$ - La société face au racisme. $79 \mathrm{~F}$

№ 10 - Le masculin

$79 \mathrm{~F}$

$\mathrm{N}^{\circ}$ 7.8 - La vérité

$99 \mathrm{~F}$

$\mathrm{N}^{\circ} 6$ - Les manipulations

$79 \mathrm{~F}$

№ 5 . La rumeur

$79 \mathrm{~F}$

No 3-4 - La transmission

$99 \mathrm{~F}$

№ 2 - Penser/classer

$79 \mathrm{~F}$

$\mathrm{N}^{\circ} 1$ - La science face au racisme $79 \mathrm{~F}$

Abonnements : pour 4 numéros France et DOM/TOM : $290 \mathrm{~F}$ - Etranger : $320 \mathrm{~F}$ (jusqu'au 30/4/89).

Les 15 premiers numéros : 599 F (au lieu de 1.067 F) jusqu'au 30/4/89).

Le genre humain - Editions du Seuil - Service des abonnements, B.P. 29, 91162 Longjumeau Cedex - Tél. 69.09.24.09. 


\title{
L'homme et la société
}

\author{
Revue internationale \\ de recherches et de synthèses en sciences sociales
}

№ 90 - Nouvelle série

XXII ${ }^{e}$ année

$4^{e}$ trimestre 1988

\section{Sommaire}

\section{Le temps et la mémoire aujourd'hui}

Louis MOREAU de BELLAING, Présentation

Gérard NAMER, Affectivité et temporalité de la mémoire

Picrrc ANSART, La pluralité des temps chez les théoriciens socialistes (1820-1870)

Isabelle BERTAUX-WIAME, Des formes et des usages des histoires de famille

Claudine ATTIAS-DONFUT, La notion de génération. Usagcs sociaux et concept sociologique

Emmanucl GARRIGUES, Le temps et la durée dans la pratique des techniques d'étude du terrain

Maria Rosa de MADARIAgA, L'image et le retour du Muurc dans la mémoire collective du pcuplc cspagnol et la guerre civile de 1936
Alain MANVILle, Ulysses SANTAMARIA, Temps, histoire et révolution
Jcan CHESNEAUX, Le temps de la modernité
Informations, Notes Critiques et Comptes rendus.

Direction-Rédaction : Institut Charles V, 10, ruc Charles V, 75004 Paris.

Directeur de la publication : René GALLISSOT.

La revue est publiée avec le concours du Centre National des Lettres.

Abonnements : Un abonnement annuel couvre 3 numcéros dont 1 numéro double.
Nouvelle sćrie : France $200 \mathrm{~F}$, Etranger $220 \mathrm{~F}$, Etranger par avion $250 \mathrm{~F}$.
Prix du numéro double : $95 \mathrm{~F}+$ Port $11 \mathrm{~F}$
L'Harmattan, 7, rue de l'Ecole-Polytechnique, 75005 Paris (Diffusion - Abonnements). 


\section{RAISON PRÉSENTE}

Directeur : Victor Leduc

$$
N^{\circ} 90 \text { - avril-mai-juin } 1989
$$

TIERS MONDE

Un statistre presage ......................................................... 3

La dette n'est pas le fin mot de I'histolre. Susan George.......... 5

Le développement et la question culturelle. Samir Amin...... 15

La démocratle et le développement. Ahmet Insel .................... 31

Faut-1l souhalter un monde èclaté ?, Serge Latouche............. 45

Une démocratle au Brésıl, Henrt Valot ................................. 57

Europe et tlers monde : des relations conflictuelles.

Guy Labertit et Bernard Ravenel ......................................... 61

L'incontournable développement. Thlerry Paquot................ 67

Drolts de l'homme et dèmocratle. $M$. Husseln

(bonnes feulles) ................................................................... 77

Le mysticisme. avatar de la modemitè. D. Paquette .............. 93

Où l'on en revient toujours au sujet. Christian Ruby .............. 101

Une lettre d'André Comte-Sponville................................ 108

Autour de la science. Michel Rouzé ................................ 109

Éducation. Enseignement, J. Placere .............................. 115

Cinéma, Alain Malassınet ............................................ 118

Théâtre, Guy Brult ........................................................ 121

Du côtė des mèdias. Maurice Audebert ............................... 126

Les livres. Frédëric Darmau. Christlan Ruby. Jeanne

Brunschwig. Henri Valot. Gabriel Gohau ............ 133

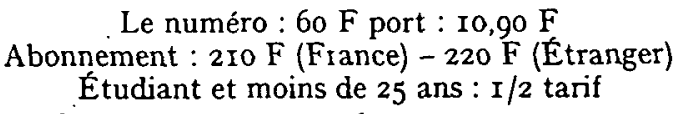

RAISON PRÉSENTE, I4 rue de l'École Polytechnique 75005 PARIS 
Nr.4,Spring1989

Frederick N. Bohrer: Assyria as Art: A Perspective on the Early Reception of Ancient Near Eastern Artefacts. Thomas C. Patterson: Political Economy and a Discourse Called "Peruvian Archeology". Per Ohrgaard: Nazism and National Identity: a Current Issue in West Germany. Inger Sjorslev: On the Edge of the Text: Three Books on Afro-Brazilian Religion. Jonathan Friedman: The Consumption of Modernity. A Review.

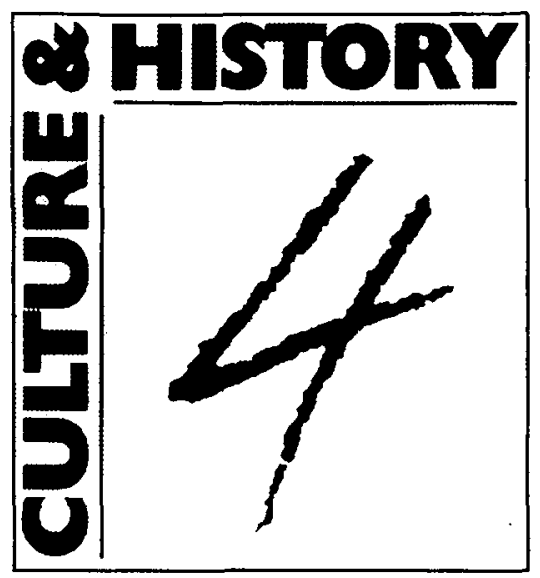

Price - single issues $\$ 20$

Culture \& History is an interdisciplinary journal dedicated to the study of historical anthropology and intellectual history. It is published by the Centre for Research in the Humanities, Copenhagen University.
Akademisk Forlag

Store Kannikestræde 6-8

POB 54

DK-1002 Copenhagen $\mathrm{K}$

Denmark

\section{CONGRES ET COLLOQUES}

Lacordaire, son pays, ses amis et la liberté des ordres religieux.
Institut de France, 1 or et 2 décembre 1989 .

- Informations : Colloque Lacordaire, 29, boulevard Latour-Maubourg, 75340 Paris Cédex 07.

10 Congrès de l'Association Internationale d'Histoire économique, Louvain, 20-24 août 1990.

- Informations et brochure détaillée : Association Internationale d'Histoire économique, B.P. 74 - B 3000 Louvain, Belgique. 
Revue Inlernatlonale consacrée ou marxisme publiée aux Presses Unlversltaires de France (P.U.F.) avec le concours du Centre Natlonal de Recherche Sclentiflque (C.N.R.S.) el de l'Université de Paris-X

Deux numéros de 208 pages par an. Interdisciplinaire et critique. Discute les analyses théoriques fondamentales et les problématiques nouvelles. Analyse la littérature marxiste mondiale, philosophie, economie, histoire, sciences sociales, littérature. Dans chaque numéro. 30 ouvrages recencés.

\section{N03 LE SOCIALISME ET L'OCCIDENT N०4: MARXISME ITALIEN \\ 1989 N'5 : LIBERALISME, ETAT DE DROIT N6 : L'URSS DE GORBATCHEV}

Rédaction : Balibar, Bidet, Barrère, Bertrand, Cotlen, Delaunay, Fogel, Hincker, Labica, Lecercle. Löwy, Quiniou, Texier, Tosel.

Abonnement : $160 \mathrm{~F}$, adresser à PUF, 14, av. du Bois-de-l'Epine, 8P9O, 91003 Evry Cédex, Tel (I) 60.77 .82 .05 .

N'4. Marxisme italien, Quelle identite ?

J. Rony. Du compromis historique à l'alternative démocratique

C. Preve, De l'eurocommunisme à l'eurogauche

G. Prestlpino, Orientations de la recherche marxiste en lialie

P. Serra, Le "Revisionnisme socialiste". 1976-1981

J.-P. Potler, Les économistes italiens et la théorie de Marx

G. Oldrinl, Labriola et les origines du marxisme italien

U. Cerronl, Marx el la science sociale

J. Texler, Rationalitê selon la fin et selon la valeur chez Gramsci

G. Markus, Le paradigme de la production et l'herméneutique

F.Hincker, Le capitalisme français et son histoire

A. Caplan et A. Lechuga, L'Etat-Providence de F. Ewald

N*5. Libéralisme, société civile, Etat de droit

D. Lossurdo, La tradition libérale et le concept universel d'homme

A. Tosel, L'Elat de droit, figures et problèmes

J. Chevaller, Le mirage de la socitété civile

J. Texier, Sur les sens de "sociêté civile" chez Gramsci

H. Hamon, Les économistes néo-libéraux

Y. Qulniou, Hayek, les limites d'un défi

M. Fleurbaey, Nozick, la théorie de l'Etat minimal

S. Petrucclant, Neo-contractualisme el réforme sociale

J. Bidet, La forme contrat, du libéralisme au socialisme

A. Heller, Marx el la modernité

M. Maidan, Le retour de Moses Hess
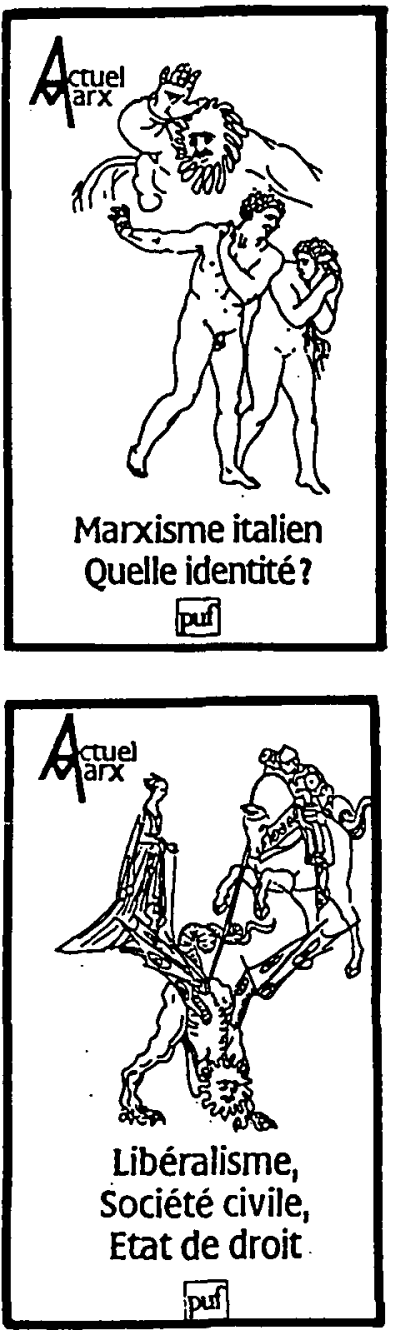


\section{societa' e storia}

anno $X I$ - n. 42 - ottobre-dicembre 1988

Giuliana Albini, Da castrum a città: Crema fra XII e XV secolo

Bruno Caizzi, Il corriere di Lindau

Enrico lachello, Il controllo del territorio nella contea di Mascali tra settecento e ottocento

Franco Della Peruta, L'emigrazione italiana in Australia dalle origini al 1914

\section{Orientamenti e dibattiti}

Rolando Minuti, Cultura scozzese e geografia dell'llluminismo: alcune considerazioni su studi recenti

Jean Boutier, I Libri d'oro del Granducato di Toscana (1750-1860). Alcune riflessioni su una fonte di storia sociale

Bronislaw Baczko, Se n'è andato uno storico

Francesco M. Cataluccio, Witold Kula: uno storico atipico

Marta Herling Bianco, La transizione dal feudalesimo al capitalismo nell'opera di Witold Kula

La professione universitaria e i progressi delle scienze. Una discussione

Francesco Traniello, Punti d'accordo e qualche perplessità

Giorgio Forti, Reclutamento del personale e carriera scientifica nel Consiglio nazionale delle ricerche

Beni culturali e organizzazione della ricerca

Francesca Boris, La memoria di una famiglia: l'archivio privato Malvasia della Serra Gabrielli

Schede, a cura di: Guido Abbattista, Federica Ambrosini, Franco Angiolini, Gianluca Battioni, Francesco Battistini, Marco Beretta, Luciano Casali, Luisa Chiappa Mauri, Salvatore Ciriacono, Luisa Dodi, Ferdinando Fasce, Alessandra Ferraresi, Luigi Ganapini, Angelo Gaudio, Dario Generali, Gaetano Greco, Nicola Lisanti, Marco Meriggi, Giuseppe Poli, Ornella Ponzellini, Saverio Russo, Giulio Sapelli, Marco Soresina, Miriam Turrini, Paola Vismara. Sono segnalati lavori di: B. Bailyn, B.A. Bembridge, M. Calegari, G. Cengiarotti, F. Dagognet, E. Decleva, R. De Felice, L. Donvito, S.R. Epstein, C. Funk, G. Gallo, J. Graunt, I.V. Hunt, M.G. Knox, N. Labanca, M. Lenci, A.W. Lovett, M. Morineau, R. Quartararo, M. Rubio Cabeza, A. Rupert Hall, D. Sacco, I. Saz Campos, F.M. Snowden, G. Sorge, E.W. von Tschirnhaus, G.S. Wood, e inoltre: Giovambattista Melloni agiografo; Inventario di archivio dell'Osservatorio astronomico di Brera; Un modello catalografico per l'archeologia industriale; Le Officine Bosco di Terni; Una parrocchia salentina in epoca moderna; Periodici italiani d'antico regime; Repertorio bibliografico di storia dell'educazione

Riviste estere

Libri ricevuti

Trimestrale - abbonamento: Italia L. 65.000 - estero L. 80.000 da versare sul c.c.p. n. 17562208 intestato a FAE Riviste srl Milano 


\section{Theory and Society}

\section{Renewal and Critique in Social Theory}

\section{Editors}

Rod Aya, University of Amsterdam; Paul DiMaggio, Yale University; Anthony Giddens, King's College, Cambridge University; Martin Jay, University of California; Jerome Karabel, University of California; Charles Lemert, Wesleyan University; Charles Tilly, New School for Social Research; Sharon Zukin, Brooklyn College; Janet Gouldner (Executive Editor), Washington University, St. Louis; Robert K. Merton (Special Advisory Editor), Columbia University; Cornelis Disco (Associate Editor), Delft University of Technology; Frank Parkin (Associate Editor), Oxford University; James C. Scott, Yale University; Karen Gall Lucas (Managing Editor), Washington University, St. Louis

Theory and Society having been founded by Alvin $W$. Gouldner is a forum for an emerging worldwide community of social theorists. Its pages are open to all those working in the advancing edges of social theory: critical theory, phenomenological sociology, the neo-Marxisms, comparative, historical and macroscopic sociology, structuralism and linguistically sensitive sociology.

Committed to preventing their mutual isolation, Theory and Society endeavours to promote clear discourse and pointed dialogue among them. This editorial view based itself on the critique of the traditional history and logic of science - a critique associated with Lakatos, Kuhn and Feyerabend, who stress the importance of comparing divergent programs.

\section{Subscription Information}

ISSN 3404-2421 1989, Volume 18 (6 issues)

Institutional rate: Dfl. $284.00 /$ US $\$ 139.00 \mathrm{incl} . p \& h$ Private rate: Dfl. $98.00 /$ US\$ $46.00 \mathrm{incl}$. p\&h

Private subscriptions should be sent direct to the publishers

Back volume information is available upon request

P.O. Box 322, 3300 AH Dordrecht. The Netherlands

P.O. Box 358, Accord Station. Hingham. MA 02018-0358, U.S.A.

KLUWER

ACADEMIC PUBLISHERS

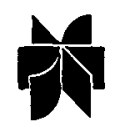




\section{techniques GQuculture}

Pour une ethnologie de l'acte traditionnel efficace

\section{REVUE SEMESTRIELLE}

Comité de patronnage Lucien Bernot. André-G. Haudricourt Andre Leroi-Gourhan $\dagger$, Axel Steensberg

\section{Secrétaire général Robert Cresswell}

Numéro 12 juillet-décembre 1988

Symboles et procès techniques

Y. Delaporte

S. Desrosiers

T. Ingold

I. Leblic

C. Méchin

S. Sadler

\section{Comptes-Rendus}

$N^{\circ} s 1$ et 2, 1983

$\mathrm{N}^{\circ} 3$ janv.-juin 1984 $N^{\circ} 4$ juil.-déc. 1984

$\mathrm{N}^{\circ} \mathrm{S} 5$ et 61985 $N^{\circ} 7$ janv.-juin 1986 $N^{\circ} 8$ juil.-déc. 1986 $N^{\circ} 9$ janv.-juin 1987 $\mathrm{N}^{\circ} 10$ juil.-déc. 1987 $N^{\circ} 11$ janv.-juin 1988

\section{Sommaire}

Les costumes du sud de la Laponie : organisation et désorganisation d'un système symbolique

Les techniques de tissage ont-elles un sens? Une proposition de lecture des tissus andins

Tools, Minds and Machines : an excursion in the philosophy of technology

De l'évolution des techniques de pêche enNouvelle-Calédonie Les débuts de la congélation dans les campagnes du Nord-Est de la France : l'expérience singulière des congélateurs collectifs Le moulin hydraulique à conduites forcées de Karapinar (Turquie)
Actes de la table ronde "technologie culturelle"(1982)

Des choses dont la recherche est laborieuse"

Aspects des agricultures insolites de l'Amérique indienne Les langages du vêtement

Par où passe la technologie l et II

De l'Himalaya au Haut Atlas, de l'Asir aux Andes

Techniques moyen-orientales

Des idées pour observer

D'autres idées pour observer

Persistances et innovations

\begin{tabular}{|c|c|c|c|}
\hline \multicolumn{4}{|c|}{ CONDITIONS DE VENTE } \\
\hline & & Abonnement & 1989 \\
\hline France & $\begin{array}{l}\text { Particulier } \\
110 \mathrm{~F} \\
90 \mathrm{~F} \text { (Etud.) }\end{array}$ & $\begin{array}{l}\text { Institution } \\
150 \mathrm{~F}\end{array}$ & \\
\hline Etranger & $130 \mathrm{~F}$ & $180 \mathrm{~F}$ & \\
\hline S'adresser à & \multicolumn{3}{|c|}{$\begin{array}{c}\text { Techniques et culture. } 27 \text { ne Paul Bert, 94204 IVRY Cedex - tél. (1) } 49604036 \text {. } \\
\text { Libeller les cheques à l'ordre de MSH/Techniques et culaure }\end{array}$} \\
\hline
\end{tabular}

vente au numéro - prix au numéro $65 \mathrm{~F}$

S'adresser au CID, 131 Bd Saint-Michel 75005 Paris, tel..(1) 43544715.

Libeller les cheques à l'ordre du CID

EDITIONS DE LA MAISON DES SCIENCES DE L'HOMME 


\section{IMPERIALISM, COLONIALISM AND THE COLONIAL AFTERMATH}

In the academic year I990-9r, the subject of the Seminar of the Shelby Cullom Davis Center for Historical Studies will be IMPERIALISM, COLONIALISM AND THE COLONIAL AFTERMATH.

The Davis Seminar invites applications for fellowships or proposals for papers on any aspect of the theme of imperialism, colonialism and the colonial aftermath. The time frame and geographical range envisaged are wide-ranging, from ancient empires to the twentieth century. The Center hopes to encourage new thinking on the forms and legitimations of empire, colonial domination, neo-colonialism, and resistance to domination; on the character of the encounters between imperial country and colonized region; and on the diverse consequences of imperialism for the dominating country and the subordinate one, including the subsequent relations of ex-colonial populations with the mother country.

Scholars looking at such issues from the perspective of cultural history are urged to apply as well as those focussing on politics, religion, law and medicine; on the conceptualization and relations of lineage groups, class, gender, and race; and on markets and economic organization. Comparative perspectives, both substantive and interpretive, are welcome. Attention will be devoted during the year to the question of historical writing about colonialism: what kinds of history have been given to colonial peoples? what kinds of history can be written about colonies and ex-colonies today?

Inquiries and requests for Fellowship Application Forms should be addressed to the Secretary, Shelby Cullom Davis Center for Historical Studies, I29 Dickinson Hall, Princeton University, Princeton, N.J. 08544-IOI7, U.S.A. The deadline for applications and letters of recommendation for r990-9I is December I, I989. Scholars who would like to offer a paper to the Seminar are asked to write to Natalie Z. Davis, Director r990-94. 


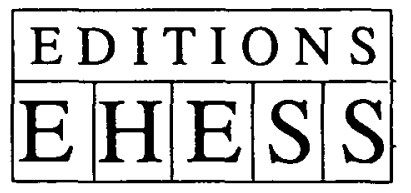

Editions de l'tecole des Hautes Études en Sciences Sociales

\section{Jürgen KOCKA \\ Les employés en Allemagne 1850-1980 Histoire d'un groupe social}

Le clivage ouvriers-employés, souvent évoqué, s'est toujours avéré extrêmement difficile à expliquer de façon systématique. Pour Jürgen Kocka, seule l'analyse historique permet de répondre à une telle interrogation. Il montre comment, à partir du milieu du $19^{\complement}$ siècle, des processus économiques, sociaux, culturels, et surtout politiques contribuent à l'émergence d'une catégorie spécifique des employẻs, qui est transversale aux secteurs économiques et aux professions, et qui s'oppose à celle des ouvriers. Centrée sur les employés du secteur privé, son étude va des débuts de l'industrialisation à la période contemporaine, et la perspective internationale à laquelle il recourt à l'occasion met bien en évidence la spécificité de la situation allemande, notamment dans la représentation des liens entre l'émergence de cette "nouvelle classe moyenne" et la montée du nazisme.

1988. 220 p. Recherches d'histoire et de sciences sociales 34 $190 \mathrm{~F}$

DIFFUSION

CID

131 bd Saint-Michel, 75005 Paris 


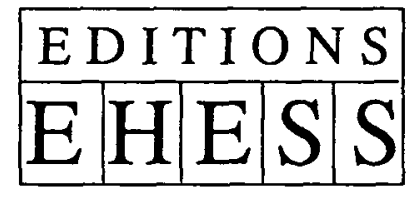

Éditions de l'Ecole des Hautes Études en Sciences Sociales

\section{Lucia CARLE \\ L'identité cachée}

\section{Paysans propriétaires dans l'Alta Langa aux XVII ${ }^{e}$-XIX ${ }^{e}$ siècles}

Pourquoi les habitants de l'Alta Langa ont-ils aussi fortement conscience d'appartenir à une région qui n'a jamais eu de réalité administrative ni politique? Les Langhe, aux limites sud du Piémont, constituent une sorte de non-région, valorisée par la littérature et le tourisme mais dont les frontières, mobiles, n'ont pas coïncidé, dans le passé, avec une entité politique : elles ne se sont fixées, et avec peine, qu'à une date toute récente. Leur identité n'est donc pas donnee : elle est une construction historique, qui reste encore inachevée, ouverte.

L'analyse de ce processus, suivi sur plusieurs siècles, fait apparaittre une dynamique plurielle qui tranche sur notre représentation spontanée de ce qu'est une société paysanne. Dynamique des espaces, dynamique des temps ; car la "cassure" n'est pas seulement celle provoquée par l'industrialisation, qui date d'hier. Dans la longue durée des $\mathrm{XVI}^{\mathrm{e}}-\mathrm{XX}^{\mathrm{e}}$ siècles, chaque village est relié aux autres par un jeu changeant d'intérêts communs. Les documents témoignent de ces transformations que la tradition orale confirme parfois ou qu'elle a, le plus souvent, oubliés.

Un modèle culturel précis et complexe se cache sous les clichés litteraires ou autres qu'évoquent aujourd'hui les Langhe. Il est l'identité, faussement évidente, en fait secrète, de cette terre et de ses habitants, et que l'auteur a cherché à pénétrer.

1989. 268 p. Recherches d'histoire et de sciences sociales 37

\section{DIFFUSION}

CID

131 bd Saint-Michel, 75005 Paris 


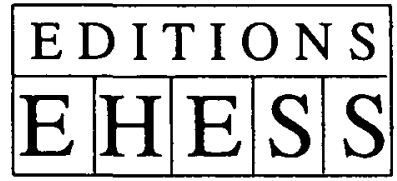

Éditions de l'École des Hautes Etudes en Sciences Sociales

\section{Marie-Vic OZOUF-MARIGNIER \\ La formation des départements}

\section{La représentation du territoire français à la fin du $18^{e}$ siècle}

Le département a deux cents ans. Il est né d'une immense ambition, réalisée en quelques mois : aménager l'espace français selon les extgences de la raison. Peu de projets témoignent davantage de la volonté qui anime les hommes de 1789 , d'une rupture radicale avec l'ancien ordre politique et social.

Entreprise de la première heure révolutionnaire, la formation des départements est fondée sur un postulat simple : de la division adéquate du territoire dépendent le bonheur des hommes et l'avènement de la société idéale. Reste à traduire cette conviction en principes et à confronter les principes à la réalité. Sur les modalités concrètes du découpage, le dẻbat s'enflamme aussitôt. En proposant une révolution territoriale, le projet rend soudain sensible la force de l'ancienne organisation de l'espace. Les habitudes, mais aussi les intérêts entrent en scène. La discussion s'en trouve compliquée, mais aussi enrichie. Car aux auteurs d'innombrables pétitions, aux membres des délégations qui harcèlent l'Assemblée, il faut trouver des arguments pour convaincre. Le livre de Marie-Vic OzoufMarignier analyse les logiques multiples. souvent rivales, qui sont au fondement d'une intervention majeure sur le territoire. Il reconstruit le jeu des représentations sur lequel s'appuie, à un moment-clé de l'histoire de la France, la pensée des rapports entre espace et pouvoir.

1988. 364 p. Recherches d'histoire et de sciences sociales 36

$176 \mathrm{~F}$

DIFFUSION

CID

131 bd Saint-Michel, 75005 Paris 


\section{CAHIERS DES ANNALES}

En vente chez ARMAND COLIN Éditeur, 103, boulevard Saint-Michel, 75240 PARIS Cedex 5

M. BLOCH, Seigneurie française et manoir anglais, $2^{\mathrm{e}}$ édition.

M. AGULHON, Le cercle dans la France bourgeoise (1810-1848).

P. CHAUNU, Histoire quantitative, histoire sérielle.

W. G. L. RANDLES, De la terre plate au globe terrestre.

S. L. KAPLAN, Le complot de famine : histoire d'une rumeur au XVIIle siècle.

R. HALÉVI, Les loges maçonniques dans la France d'Ancien Régime.

R. E. GIESEY, Cérémonial et puissance souveraine. France, XVe.XVIIe siècles.

La revue ANNALES ESC est disponible et peut être commandée chez les libraires suivants :

Aix-en-Provence (Librairie de l'Université)

Besançon (Camponovo)

Bordeaux (Mollat)

Caen (J.-C. Marie)

Dijon (Librairie de l'Université)

Grenoble (Arthaud, FNAC, Librairie de l'Université)

Lille (Meura, Furet du Nord)

Lyon (Flammarion, Nouveautés, La Proue)

Marseille (Maupetit)

Montpellier (Brèche, Sauramps)

Nantes (Vent d'Ouest)

Nice (FNAC)

Paris (Librairie du Regard, Interférences, La Procure, Lavocat, Escalier, Un Moment, Picard, Temps retrouvé, Autrement dit, Boutique des Cahiers, SNC Cluny Sorbonne, FNAC Montparnasse, P.U.F., FNAC Forum)

Pau (Tonnet)

Poitiers (Librairie de l'Université)

Strasbourg (Berger Levrault, Librairie des Facultés, FNAC, Kléber)

Toulouse (Librairie des Facultés, Privat)

Tours (Boîtes à Livres)

Pour les années 1929 à 1976, ainsi que pour les Index analytiques 1929-1948 et 1949-1968, s'adresser à KRAUS REPRINT, Route 100, Millwood, New York 10546, USA. 